\title{
Visual working memory simultaneously guides facilitation and inhibition during visual search
}

\author{
Blaire Dube $^{1} \cdot$ April Basciano $^{1} \cdot$ Stephen M. Emrich $^{2} \cdot$ Naseem Al-Aidroos $^{1}$
}

Published online: 7 April 2016

(C) The Psychonomic Society, Inc. 2016

\begin{abstract}
During visual search, visual working memory (VWM) supports the guidance of attention in two ways: It stores the identity of the search target, facilitating the selection of matching stimuli in the search array, and it maintains a record of the distractors processed during search so that they can be inhibited. In two experiments, we investigated whether the full contents of VWM can be used to support both of these abilities simultaneously. In Experiment 1, participants completed a preview search task in which (a) a subset of search distractors appeared before the remainder of the search items, affording participants the opportunity to inhibit them, and (b) the search target varied from trial to trial, requiring the search target template to be maintained in VWM. We observed the established signature of VWM-based inhibition-reduced ability to ignore previewed distractors when the number of distractors exceeds VWM's capacity-suggesting that VWM can serve this role while also representing the target template. In Experiment 2, we replicated Experiment 1, but added to the search displays a singleton distractor that sometimes matched the color (a task-irrelevant feature) of the search target, to evaluate capture. We again observed the signature of VWM-based preview inhibition along with attentional capture by (and, thus, facilitation of) singletons matching the target template. These findings indicate that more than one VWM representation can bias attention at a
\end{abstract}

Blaire Dube

bdube@uoguelph.ca

1 Department of Psychology, University of Guelph, Guelph, Ontario N1G 2W1, Canada

2 Department of Psychology, Brock University, Catharines, ON, Canada time, and that these representations can separately affect selection through either facilitation or inhibition, placing constraints on existing models of the VWM-based guidance of attention.

Keywords Visual search · Visual working memory . Inhibition

Visual search - the process of trying to find one visual item amid distractors - can be a complex and attention-demanding task, yet it is one we regularly perform with a high level of efficiency (Wolfe, 1994). In some instances, the efficiency of search is supported by visual working memory (VWM), a short-term memory system that can represent a small amount of visual information equivalent to about three or four visual objects (Luck \& Vogel, 1997). For example, VWM can be used to store the identity of the stimulus you are looking for: the target template (Carlisle, Arita, Pardo, \& Woodman, 2011; Woodman \& Arita, 2011). Notably, a by-product of storing a target template in VWM is that search is guided toward stimuli sharing the template's features (Bundesen, 1990; Desimone \& Duncan, 1995; Duncan \& Humphreys, 1989; Olivers, Meijer, \& Theeuwes, 2006; Woodman \& Luck, 2007; Woodman, Luck, \& Schall, 2007). Beyond supporting this guidance, VWM also improves the efficiency of search by maintaining a record of processed distracting information in a search display so that this information can be subsequently ignored, known as visual marking (Al-Aidroos, Emrich, Ferber, \& Pratt, 2012; Watson \& Humphreys, 1997). Thus, VWM contributes to visual search via two distinct processes: It facilitates the processing of stimuli resembling the target template, and inhibits previously evaluated distractors to prevent them from being reselected. Can VWM support both processes simultaneously? Such an ability would have implications for models 
of how VWM guides attentional selection, and in particular the ongoing debate about whether guidance is limited to a single item in memory (Houtkamp \& Roelfsema, 2006; Olivers, Peters, Houtkamp, \& Roelfsema, 2011), or whether the full contents of VWM can be used to bias attention (see Beck, Hollingworth, \& Luck, 2012, for evidence in support of this view). Thus, in the present study we aimed to identify whether or not VWM can be used to simultaneously support processes of facilitation and inhibition.

\section{VWM-based facilitation during visual search}

The ability to guide search on the basis of knowledge of the search target has long been a core component of theories of visual search (Bundesen, 1990; Duncan \& Humphreys, 1989; Wolfe, 1994). Moreover, there has long been evidence that, at least for some visual searches, this guidance is accomplished by representing the search template within VWM (for a review, see Soto, Hodsoll, Rotshtein, \& Humphreys, 2008). For example, single-unit recordings in monkeys during the delay period between when a search target is specified and when the search display is presented have revealed increased firing of inferotemporal neurons that preferentially respond to the target feature, and more vigorous firing of these already active neurons when the target later appears in the search display (Chelazzi, Duncan, Miller, \& Desimone, 1998; Chelazzi, Miller, Duncan, \& Desimone, 1993). This pattern is consistent with a biased-competition model of attention, in which higher-level representations (i.e., in working memory) bias selection through a top-down influence on activity within the perceptual cortices (Desimone \& Duncan, 1995). Here, the representation of the target template in VWM guides selection toward the search target by facilitating perceptual activity associated with stimuli resembling the template so that these stimuli are selected over the competing signals produced by search distractors.

More recently, the emphasis of research in this field has been to use behavioral and event-related potential (ERP) measures in humans to investigate the search conditions under which this guidance occurs (Dalvit \& Eimer, 2011; Olivers, 2009; Olivers et al., 2006; Soto, Heinke, Humphreys, \& Blanco, 2005). Consistent with the facilitatory VWM-based effects observed by Chelazzi and colleagues (1998; Chelazzi et al., 1993), search distractors capture attention (i.e., increase search times) to a greater extent when they resemble the content of VWM representations (Olivers et al., 2006; Soto et al., 2008). Of most relevance to the present study are investigations using ERPs that have clarified when target templates are stored in VWM and when other memory systems are used, such as long-term visual memory (Carlisle et al., 2011; Woodman \& Arita, 2011). Using the contralateral delay activity (CDA) as an index of VWM activity (Vogel \& Machizawa,
2004), Carlisle and colleagues demonstrated that the contribution of VWM in the maintenance of the target template dissipates quickly during variable searches, in which the identity of the target changes across trials. Specifically, they found that the CDA can be observed after participants first learn the identity of a new search target, but not after the search target repeats for about seven trials. This pattern provides converging evidence of a role for VWM in maintaining the target template and clarifies that VWM only meaningfully contributes throughout the duration of variable searches, in which the target changes each trial, and not static searches, in which the target remains the same.

\section{VWM-based inhibition during visual search}

Beyond the facilitation afforded by VWM-based search templates, VWM can also guide inhibitory processes during search (Al-Aidroos et al., 2012; Belopolsky \& Theeuwes, 2009; Brockmole \& Henderson, 2005; Castel, Pratt, \& Craik, 2003; Downing \& Dodds, 2004; Emrich, Al-Aidroos, Pratt, \& Ferber, 2009, 2010; Sawaki \& Luck, 2011; Theeuwes, Olivers, \& Chizk, 2005; Woodman \& Luck, 2007). For example, as stimuli are identified as distractors during a search, VWM maintains a record of these distractors so that they can subsequently be ignored. To demonstrate this role, Emrich and colleagues (2010) investigated the consequences of a concurrent VWM load on search performance, which should interfere with inhibition and slow search. Because VWM is capacity-limited, the effect of a concurrent load will vary depending on whether or not the number of search distractors exceeds the putative three- to four-item capacity of memory ${ }^{1}$ (see Emrich et al., 2010, Fig. 1). Consistent with these predictions, Emrich and colleagues tested search set sizes above capacity and observed increases in overall search times following increases in the concurrent VWM load, until load reached the capacity of memory.

Further evidence for VWM-based inhibition has come from the preview search paradigm (Watson \& Humphreys, 1997). During preview searches, a subset of the search distractors are revealed to the participant before the rest of the search display. Accordingly, this paradigm allows direct control over the amount of information that participants should be inhibiting at the time that search begins. Although

\footnotetext{
${ }^{1}$ Emerging resource models have challenged the three- to four-item limit by suggesting that, rather than a discrete item limit, VWM storage is limited by a resource pool shared dynamically among items (Bays, Catalao, \& Husain, 2009; Bays \& Husain, 2008; Keshvari, van den Berg, \& Ma, 2013). Regardless of the number and quality of items that individuals can remember in VWM tasks, when observers use VWM of simple visual stimuli to guide behavior, the effect is limited to about three or four visual objects (Al-Aidroos et al., 2012; Alvarez \& Cavanagh, 2004; Emrich, Ruppel, Al-Aidroos, Pratt, \& Ferber, 2008).
} 
a

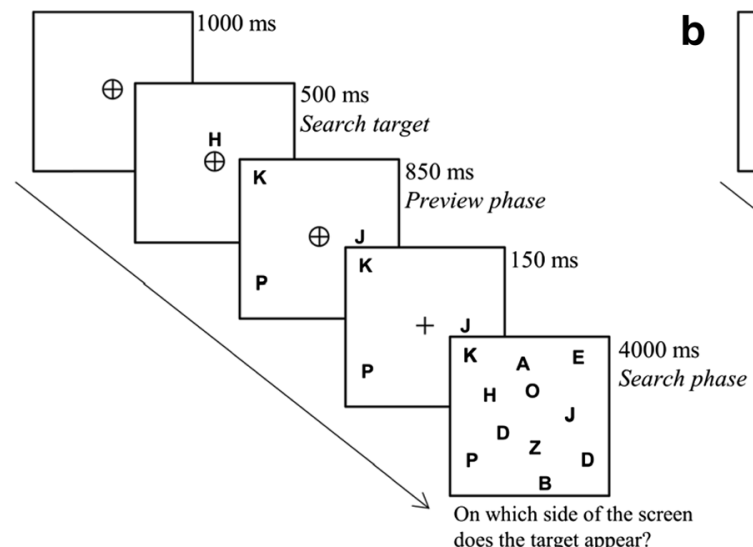

b

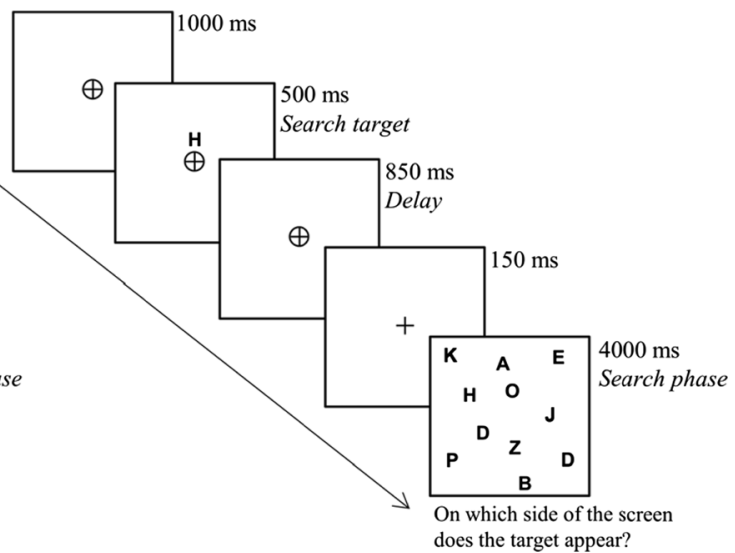

Fig. 1 Trial sequences in Experiment 1 for (A) preview trials, and (B) no-preview trials. The actual stimuli were green letters on a black background.

numerous mechanisms support preview effects, through both the inhibition of previewed items and the facilitation of nonpreviewed items (for reviews, see Donk, 2006, and Olivers, Humphreys, \& Braithwaite, 2006), Al-Aidroos et al. (2012) reported a task that adequately isolates the contribution of VWM-based inhibition, so that the capacity for inhibition can be quantified and compared against VWM. In this study, they had participants search for the letter " $H$ " among a number of nonpreviewed distracting letters, and also added between one and seven previewed distractors to the search (see Fig. 1 below for a similar design). To the extent that the previewed distractors are successfully inhibited, each additional distractor should have little impact on search times. Put differently, the effectiveness of preview inhibition in this paradigm can be measured as the search slope across the number of preview distractors: More effective preview inhibition will result in shallower search slopes. Consistent with a role for VWM in contributing to the inhibition of previewed distractors, Al-Aidroos and colleagues observed that preview inhibition was more effective when the number of previewed distractors was below (zero to three) than when it was above (four to seven) the capacity of VWM: The search slope across preview set sizes 0 to 3 was close to $0 \mathrm{~ms} /$ item, and the slope across preview set sizes 4 to 7 was equivalent to the slope of nonpreview searches. Building off of this finding, AlAidroos and colleagues observed that individual differences in preview inhibition capacity correlated with individual differences in VWM capacity, and that the capacity of preview inhibition was specifically tied to the number of inhibited distractors, rather than to other factors, like search difficulty. This study provided direct converging evidence for VWM-based inhibition during search and established a paradigm for measuring this contribution of memory separately from the contributions of other preview mechanisms.

\section{The present study}

The theories and evidence discussed thus far point to two important functions of the representations in VWM: Information matching relevant representations is facilitated in search, and information matching representations that are known to be irrelevant is inhibited. Is it the case that VWM can either support the facilitation or the inhibition of matching stimuli, or can VWM support both of these processes concurrently? To the best of our knowledge, no experiment has directly tested for the operation of both roles at the same time, and current theories regarding VWM-based guidance of attention offer support both for and against each possibility. Olivers and colleagues (2011), for instance, proposed a divide in VWM that allows for relevant representations to bias attention in visual search. According to this account, items in VWM are classified into two functionally different categories: an active item that represents the target template and biases attention toward perceptually similar inputs, and remaining accessory items that are represented in VWM but do not directly influence the selection of perceptual inputs. The researchers suggested that interactions between selective attention and VWM are only evident for a single item when it is granted access to the active position: When observers are required to maintain an item in VWM while completing an intervening search task for a consistent target, a singleton in the search array matching the memory item captures attention (Dalvit \& Eimer, 2011; Olivers, 2009; Olivers et al., 2006; Soto et al., 2005); however, this capture effect disappears if the target of the visual search is variable, and thus supported by VWM (Downing \& Dodds, 2004; Peters, Goebel, \& Roelfsema, 2009; Woodman \& Luck, 2007). Consequently, it appears that VWM-based guidance in 
search is possible for only a single active representation. If only one representation can influence the selection of perceptual inputs, it may be the case that concurrent inhibition and facilitation are not possible, and that VWM can support only one of these processes for a given search.

The division in VWM proposed by Olivers and colleagues, however, inherently claims that there are separate consequences for the items placed into different states of memory. Although it has been shown numerous times that accessory items do not guide facilitation during search (Downing \& Dodds, 2004; Houtkamp \& Roelfsema, 2006; Woodman \& Luck, 2007; although see Beck et al., 2012), perhaps it is the case that accessory representations have the opposite effect. That is, whereas search is guided toward stimuli matching the active representation, search may be concurrently guided away from stimuli matching the accessory representations. Indeed, there is some evidence of the inhibition of accessory items in a visual search with a variable search target (i.e., a target supported by VWM; see Downing \& Dodds, 2004, Exp. 2), though that study failed to observe simultaneous facilitation. Thus, it remains an open question whether the full contents of VWM can simultaneously guide both facilitation and inhibition during search, and resolving this question will place constraints on contemporary models of the memorybased guidance of attention. Accordingly, in the present study we tested for simultaneous VWM-based inhibition and facilitation during search.

\section{Experiment 1}

Al-Aidroos and colleagues (2012) demonstrated how to use a preview-search paradigm to test for the contribution of VWM to inhibition during search. This previous study only employed a static search target (i.e., the letter "H"), a condition in which the search target template should be maintained in long-term memory (Carlisle et al., 2011). The primary purpose of Experiment 1 was to replicate the paradigm of AlAidroos et al. with a variable search target, to test whether the signature of VWM-based inhibition would continue to be observed when VWM was also used to maintain the target template. Participants completed blocks of preview searches with either a static target or variable targets, and we varied the number of previewed distractors. In the static condition, we should be able to replicate the results of Al-Aidroos et al.: a shallower search slope across preview set sizes below VWM's capacity (zero to three previewed items), and a steeper search slope above (four to seven previewed items). Note that here we have assumed capacity to be three, which is consistent with previous data from VWM tasks demonstrating performance decrements when the number of to-be-remembered items exceeds this limit (Cowan, 2001; Luck \& Vogel, 1997), as well as with the capacity limit assumed by Al-Aidroos et al. If, and only if, VWM can simultaneously support the target template and preview inhibition, we should observe the same pattern in the variable-search-target condition.

\section{Method}

Participants Thirty-five volunteers, $18-30$ years of age ( $M=$ $21.8, S D=2.74 ; 16$ males, three left-handed), participated for monetary compensation. The participants were members of the general community recruited through posters and online postings, and were naïve to the purpose of the experiment. All participants reported having normal or corrected-to-normal vision, provided written and informed consent, and were fully debriefed immediately following the experiment. Three of the participants were removed from the reported analyses because they responded to more than $20 \%$ of the catch trials (described below), and one participant was removed for not following instructions during the experiment, resulting in a final sample of 31 participants.

Apparatus and stimuli This experiment was conducted on a Mac computer with a 32-bit color LCD monitor, and responses were made via keyboard buttonpress on an Apple keyboard at a viewing distance of approximately $50 \mathrm{~cm}$. All displays were presented at a resolution of 1,680 $\times 1,050$ pixels. The experiment was controlled using Java and a Windows operating system. This setup was used for all subsequent experiments. The experimental stimuli were green uppercase letters (approximately $0.5^{\circ}$ of visual angle in width and height), viewed on a black background with a white fixation stimulus $\left(0.4^{\circ} \times 0.4^{\circ}\right)$. The stimuli were presented in an array on a virtual $8 \times 8$ square grid, with adjacent grid positions separated by $2.25^{\circ}$ to encourage participants to use eye movements to complete the search task (Al-Aidroos et al., 2012). The sizes and positions of the stimuli used here should be sufficient to prevent density effects at large preview set sizes (Cohen \& Ivry, 1989, 1991; Pashler, 1987).

Design and procedure On all trials, participants performed a visual search to determine the presence of a target letter (see Fig. 1). We used a blocked design that alternated between three blocks of static searches and three blocks of variable searches, with the starting block type determined randomly. The search targets for static blocks were determined at the beginning of the experimental session by randomly selecting three letters from the English alphabet (excluding O and Q); variable search targets and search distractors for each trial were selected randomly without replacement from the remaining 21 letters. Note that for variable searches, the target was changed on every trial. The identity of the variable target was drawn randomly on each trial. On-screen instructions were presented at the beginning of each block to indicate the type of target that would be used (i.e., static or variable). 
Within every block there were two types of search trialspreview and no-preview trials, both consisting of three phases. First, a target cue was presented $1^{\circ}$ above the fixation stimulus for $500 \mathrm{~ms}$, informing the participant of the search target for the current trial, followed by a 1-s delay. Next, a 1-s preview phase occurred during which some of the search distractors could be previewed. On no-preview trials, this phase contained only the fixation stimulus, and on preview trials, one, two, three, four, five, or seven search distractors were presented. Participants were informed at the beginning of the experiment that they should ignore these letters, as they would remain visible during the search and would never include the search target. Shortly before the end of the preview phase (150 ms), the circle was removed from the fixation stimulus to alert participants that the next phase of the experiment was about to start. The final phase was the search phase. Either eight distractors were added to the display (on catch trials), or seven distractors and one target. On no-preview trials, eight, 11 , or 15 distractors were added to equate the total set sizes with some of the preview conditions (i.e., preview conditions with no, three, or seven previewed items, plus search distractors). Participants had up to $4 \mathrm{~s}$ to report whether the target was to the left or right of fixation by pressing " $Z$ " or " /," respectively. If no target was present, participants were instructed to withhold responding. No-target catch trials were included to ensure that participants searched both sides of the display prior to responding.

Practice trials were included to familiarize participants with the task, followed by six blocks of 60 experimental trials and six catch trials (396 trials in total). The locations of search stimuli were randomized every trial. Participants performed an articulatory suppression task (reciting a string of four letters out loud) throughout each block, to minimize the use of verbal memory (Baddeley, 1986).

Three within-participants conditions were included: target type (static vs. variable), preview type (preview vs. no preview), and set size. The manipulations of set size differed across preview conditions, with seven levels for preview trials (zero, one, two, three, four, five, or seven previewed distractors, plus eight nonpreviewed distractors) and three levels for no-preview trials (eight, 11, or 15 nonpreviewed search distractors presented together, set sizes comparable to zero, three, or seven previewed distractors plus eight additional distractors in the preview condition). Trials with set size 0 were treated as both preview and no-preview trials.

It should be noted that because the variable-target condition in the present paradigm encourages participants to use VWM for two different components of the task (i.e., remembering previewed items and remembering the search target), participants were effectively performing a dual task. Under such circumstances, testing for the simultaneous performance of both tasks is complicated by the potential for task switching (i.e., using VWM to remember preview items on some trials, and search targets on others). By design, however, this experiment made task switching unlikely, since participants could only complete the variable-target search task if they remembered the identity of the search target. Consequently, to the extent that participants achieved a high level of accuracy on the search task, any evidence of VWM-based preview inhibition could be attributed to the simultaneous use of VWM for maintaining the search target and guiding the inhibition of preview items.

\section{Results and discussion}

Error trials $(3.5 \%)$ and trials on which reaction times (RTs) did not fall within 2.5 standard deviations (SDs) of the condition mean for the participant $(2.3 \%)$ were excluded from the analyses below. When describing the analyses, the number of items that were previewed in the preview condition is written in brackets next to indications of search set size. Accuracy across conditions was compared by using a 2 (Preview Type: preview vs. no preview) $\times 2$ (Set Size: 11[3] and $15[7]) \times 2$ (Target Type: static vs. variable) repeated measures analysis of variance (ANOVA). Aside from a main effect of target type, $F(1,30)=18.77, M S E=.072, p<.001$, indicating that accuracy was improved in static as compared to variable searches, and a main effect of set size, $F(1,30)=27.28, M S E$ $=.047, p<.001$, indicating that accuracy decreased as set size increased, all other main effects and interactions were not significant, $F_{\mathrm{S}}<1$, indicating that no preview-type effects or preview-type interactions were being driven by speed-accuracy trade-offs.

Visual search RTs are summarized in Fig. 2. Looking at the figure, there appears to be a preview benefit in that, overall, preview trials had faster RTs than no-preview trials, and a shallower overall search slope. Although preview effects are conventionally measured as differences in search slope (e.g., Watson \& Humphreys, 1997), our predictions about slope varied with set size, and thus we focus first on the overall changes in search RTs. To assess whether search times were statistically significantly faster in the preview than in the nopreview conditions, we restricted analyses to the set sizes common to both the preview and no-preview conditions, and compared RTs using a 2 (Preview Type: preview vs. no preview) $\times 2$ (Set Size: 11[3] and 15[7]) $\times 2$ (Target Type: static vs. variable) repeated measures ANOVA. A main effect of preview type was evident, $F(1,30)=49.72, M S E=3.21 \times$ $10^{4}, p<.001, \eta_{\mathrm{p}}{ }^{2}=.624$, indicating that participants did have significantly lower search times in the preview condition. Furthermore, we observed a main effect of set size, $F(1,30)$ $=129.81, M S E=1.36 \times 10^{4}, p<.001, \eta_{\mathrm{p}}{ }^{2}=.812$; RTs increased as a function of set size. Notably, there was no main effect of target type, nor any significant two- or three-way interactions: Target Type $\times$ Preview Type, $F(1,30)=2.69$, $M S E=2.15 \times 10^{4}, p=.112$; all other $F$ values $<1$. 
a

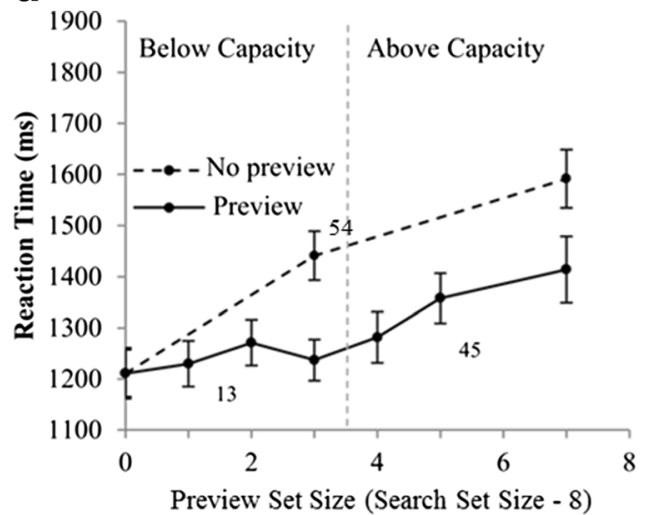

Fig. 2 Mean search reaction times in both preview and no-preview conditions when (A) the search target was static and (B) the search target was variable. For both search target types, search slopes in the preview condition are shallower for set sizes below than for those b

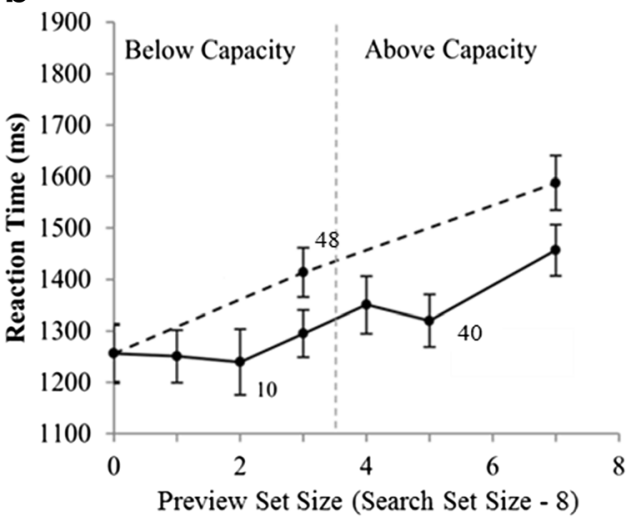

above VWM capacity. Error bars indicate $95 \%$ confidence intervals excluding between-subjects variance (Cousineau, 2005). Floating numbers represent the slopes for preview conditions below and above VWM capacity, and for the no-preview condition.
Collectively, these initial analyses align with the analyses of search slopes from past preview studies: Participants are faster to locate a target when they are able to preview a subset of the distractors in the search array. Moreover, for set sizes at or above working memory capacity, the preview advantage is consistent (i.e., visual inspection of the graph suggests that the difference between search RTs in the preview and nopreview conditions is the same at set size 13[5] as it is at set size $15[7])$.

The primary aim of Experiment 1 was to test for VWMbased preview inhibition during both static and variable searches by comparing search slopes in the preview condition across preview set sizes below $(0,1,2,3)$ versus above $(4,5$, 7) VWM capacity. Linear regression was used across the set sizes below ( 0 to 3 ) and above (4 to 7) capacity to calculate the respective slopes. In support of the conclusion that VWMbased preview inhibition occurred for both target types, a 2 (Target Type: static vs. variable) $\times 2$ (Capacity: below vs. above) repeated measures ANOVA of these search slopes revealed a significant main effect of capacity, $F(1,30)=7.4$, $M S E=4.16 \times 10^{3}, p=.011, \eta_{\mathrm{p}}{ }^{2}=.198$, and no main effect of target type or interaction, both $F$ values $<1$. As can be seen in Fig. 2, the search slopes below VWM capacity are close to $10 \mathrm{~ms} /$ item, suggesting that previewed distractors were mostly ignored. For previewed-distractor set sizes that exceeded VWM capacity, however, search slopes increased, suggesting a decrease in the effectiveness of preview inhibition. Of most interest, the significant main effect of capacity did not interact with target type, suggesting that this pattern occurred for both static and variable searches.

As a more direct investigation of this question, we used a priori one-tailed $t$ tests to test for the signature of VWM-based preview inhibition in the static and variable conditions separately. For static search targets, the search slope was significantly shallower when the number of previewed items was below VWM capacity $(M=12.67, S D=54.05)$ than when it was above $(M=44.87, S D=84.91), t(30)=-1.85$, $p=.037$. The same was true for variable search targets: The search slope below capacity $(M=9.67, S D=66.24)$ was shallower than the search slope above capacity $(M=$ $40.45, S D=65.93), t(30)=-1.81, p=.041$. These results suggest that VWM-based preview inhibition is more effective below than above VWM capacity using both a static and variable target. Given that the two roles for VWM studied in the variable-search-target condition were occurring simultaneously, it might be reasonable to predict that there could be some interference. That is, using VWM for the target template should reduce the VWM resources for preview inhibition. Through visual inspection of Fig. 1, it does appear that preview inhibition reaches its capacity around preview set size 3 for static searches, and preview set size 2 for variable searches; however, our study was not designed to address this point, and provides insufficient power to assess this difference using inferential statistics.

In summary, the finding that preview inhibition is as effective with a variable as it is with a static search target suggests that, even when VWM resources are required to maintain the identity of a search target, a VWM-based preview inhibition is still evident. Here, the inclusion of a variable-search condition allowed us to assess whether target template maintenance and distractor inhibition could operate simultaneously. Our results indeed provide support for this hypothesis. To further verify that both facilitation and inhibition were being used, we could capitalize on a central characteristic of target template maintenance by VWM: 
attentional capture by items resembling the target template. If VWM were supporting the identity of the target template without facilitating matching items in search, or if VWM were not supporting the template at all, attentional-capture effects would not be evident. We conducted Experiment 2 to investigate this point: During preview searches with a variable target, would we observe evidence for VWM-based inhibition of previewed distractors (as in Exp. 1) and, at the same time, VWM-based facilitation of stimuli that resembled the target template?

\section{Experiment 2}

The aim of Experiment 2 was twofold: to replicate the results from Experiment 1, and to test for evidence of attentional capture due to the contents of VWM. Specifically, if the target template were being stored in VWM, then any search items resembling the target template should capture attention. This prediction is based on previous studies that have demonstrated preferential processing of stimuli that resemble the contents of VWM (i.e., those that share features) as compared to those that do not (Dalvit \& Eimer, 2011; Eimer \& Kiss, 2010; Olivers, 2009; Olivers \& Eimer, 2011; Olivers et al., 2006).

To test this prediction, in Experiment 2 our participants once again performed preview searches for a specific letter; however, when the search target was specified at the beginning of each trial, it was presented using a randomly selected color, and a color singleton was added to the search display that either matched or did not match the color of the target template. Although color was irrelevant to the present task, if the target template were maintained in VWM, matching singleton distractors should produce greater attentional capture than nonmatching singletons, leading to a significantly greater slowing of search times.

\section{Method}

Participants Thirty-three volunteers, 18-34 years of age ( $M=$ 21.6, $S D=3.22 ; 15$ males, three left-handed), participated for monetary compensation. These participants were members of the general community recruited through posters and online postings, and were naïve to the purpose of the experiment. All participants reported having normal or corrected-to-normal vision, provided written and informed consent, and were fully debriefed immediately following the experiment. Three participants were removed from the reported analyses because they responded to more than $20 \%$ of the catch trials, resulting in a final sample of 30 participants.

Apparatus and stimuli Figure 3 depicts a standard trial sequence and the stimuli used in Experiment 2. The changes in
Experiment 2 were made to accommodate the manipulation of attentional capture by a colored singleton. The background was lightened to a medium gray, and stimuli were presented in seven colors (black, green, yellow, blue, red, brown, and purple). All letters were presented in boldface to elicit a stronger sensory signal, facilitating attentional capture. Otherwise, the stimuli and apparatus were identical to those used in Experiment 1.

Design and procedure This task included only the critical conditions from Experiment 1, to test for the signature of VWM-based preview inhibition: Only the preview condition was used, and only with two preview set sizes below (1 and 3) and two preview set sizes above (5 and 7) VWM capacity. All but one of the search stimuli were presented in the same color, randomly selected from the pool of seven colors. The remaining search item was presented in a different randomly selected color, and thus served as a singleton. To ensure that color was task-irrelevant, the search item presented as a singleton was selected randomly from all of the search stimuli, including the target. The results from trials with target singletons are excluded from the analyses below, as this trial type had low power and, unlike distractor singleton trials, does not provide a clear test of our hypotheses. Two within-participants conditions were created: distractor type (matching vs. nonmatching) and set size (one, three, five, or seven previewed items, plus eight additional items). Participants completed practice trials to ensure familiarity with the task, prior to completing eight blocks of 40 experimental trials and eight catch trials ( 384 trials in total). In all other respects, the design and procedure of Experiment 2 were identical to those of Experiment 1.

\section{Results and discussion}

We analyzed search accuracy as a function of distractor condition to test for distractor-related speed-accuracy trade-offs. There was no significant difference in accuracy between the matching (5.13\% incorrect) and nonmatching (5.19\% correct) color singleton conditions, $t(29)=0.13, p=.90$. For all further analyses, error trials (5.16 \%) and trials on which RTs did not fall within $2.5 \mathrm{SDs}$ of the participant's mean RT for the relevant condition $(1.58 \%)$ were excluded.

Figure 4 depicts the visual search RT results. We first tested for the signature of VWM-based inhibition by conducting a 2 (VWM Capacity: below vs. above) $\times 2$ (Distractor Type: matching vs. nonmatching) repeated measures ANOVA on the observed search slopes. As would be expected on the basis of visual inspection, we observed no main effect of distractor type on search slopes, $F(1,29)=0.83, M S E=2.88$ $\times 10^{3}, p=.37$; however, there was a significant main effect of VWM capacity, $F(1,29)=10.87, M S E=$ $4.77 \times 10^{3}, p=.003, \eta_{\mathrm{p}}{ }^{2}=.388$. Thus, VWM-based 
a

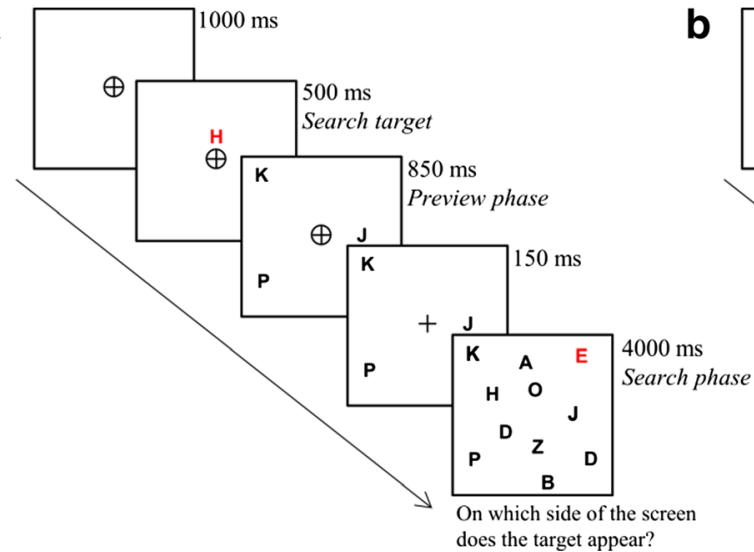

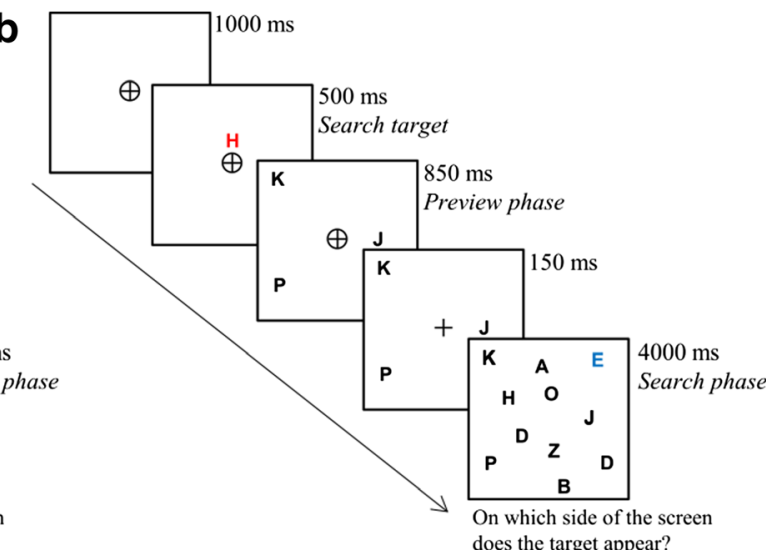

does the target appear?

Fig. 3 Trial sequences in Experiment 2 for visual searches with (A) a matching distractor and (B) a nonmatching distractor.

preview inhibition was observed for both distractor conditions. No two-way interaction was evident, $F<1$.

To specifically assess VWM-based preview inhibition for each distractor condition separately, a priori one-tailed $t$ tests compared search slopes above and below capacity. As anticipated, when the number of previewed items was below capacity, the search slopes in both the matching and nonmatching conditions were significantly shallower than when the number of previewed items exceeded capacity, $t(29)=-2.24, p=.02$, and $t(29)=-2.38, p=.01$, respectively. These findings illustrate the signature of VWM-based preview inhibition, replicating previous research as well as the results of Experiment 1.

The primary aim of Experiment 2 was to assess attentional capture of items resembling the target template. To determine whether the distractor type affected search performance, we analyzed the search RTs using a 2 (Distractor Type: matching

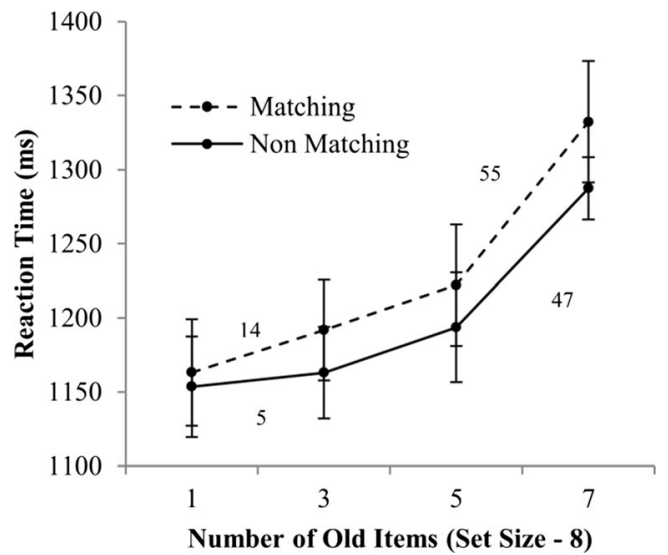

Fig. 4 Visual search reaction times (RTs) for Experiment 2. RTs are slower when the distractor in the search array matches the target template, indicating template facilitation, and search slopes are more efficient below than above VWM capacity, indicating VWM-based inhibition. Error bars indicate $95 \%$ confidence intervals excluding between-subjects variance (Cousineau, 2005). Floating numbers represent the search slopes for both distractor conditions (matching and nonmatching) below and above VWM capacity. vs. nonmatching $) \times 4$ (Preview Set Size: $1,3,5,7)$ repeated measures ANOVA. We found a significant main effect of preview set size, $F(2.3,66.2)=27.86, M S E=1.29 \times 10^{4}$, $p<.001, \eta_{\mathrm{p}}{ }^{2}=.58$, indicating longer search times for larger preview set sizes (degrees of freedom have been adjusted because we could not assume sphericity for this test). Additionally, a significant main effect of distractor type emerged, $F(1,29)=7.01, M S E=6.69 \times 10^{3}$, $p=.013, \eta_{\mathrm{p}}{ }^{2}=.17$, such that the presence of a matching color singleton impaired search performance to a greater degree than did the presence of a nonmatching color singleton. No significant interaction between preview set size and distractor type was observed, $F<1$.

In summary, Experiment 2 provides two important findings. First, it replicates the results of Experiment 1, once again suggesting that VWM-based preview inhibition occurs even in variable searches that require VWM to maintain the target template. Second, Experiment 2 offers evidence that the target template is, indeed, stored in VWM, and further that items resembling this target template are facilitated during visual search. This finding in particular falls in line with previous work by Watson and Humphreys (2005), who presented irrelevant color distractors during the preview phase. The items disrupted the preview benefit only when their color matched that of the anticipated target, suggesting that the inhibition of old stimuli occurs alongside the maintenance of an anticipatory set for new items. Here, we demonstrated simultaneous inhibition of the previewed items and facilitation of items sharing features with the target in the search phase.

\section{General discussion}

The primary purpose of the present study was to assess whether or not the contents of VWM can be used to simultaneously guide both the inhibition and facilitation 
of items during visual search. Using a preview search paradigm, we tested whether VWM can support the inhibition of a subset of previewed search distractors while also maintaining the identity of the search target, in turn guiding attention toward target-matching items. Across two experiments, we found that when participants are able to preview a subset of distractors in a search task with a variable target, the signatures of VWM-based preview inhibition and VWM-based target facilitation can both be observed-namely, more effective preview inhibition when the number of previewed items is below VWM capacity, and attentional capture by distractors that resemble the search target, respectively. Together, these results suggest that VWM can guide both facilitatory and inhibitory attentional abilities at the same time.

\section{Implications for theories of VWM-guided attention}

To reconcile the mixed evidence regarding the role of VWM contents in guiding attention (Downing \& Dodds, 2004; Olivers et al., 2006), Olivers and colleagues (2011) proposed a dual-state model of VWM that identifies when these representations will guide attention and when they will not. According to their account, the representations in VWM are divided into one of two states: active or accessory. Only those representations in the active state guide attentional selection, and only one representation may occupy the active position at a time. The data that we report here reveal additional constraints that inform our understanding of this model: In our experiments, multiple VWM representations on a given trial guided attentional selection, and further, attention was simultaneously guided toward stimuli matching the target template and away from previewed distractors in the search array.

Can our results be reconciled with the dual-state model? We do offer indirect support for a division in VWM representations: We observed that different VWM representations can have different attentional effects. Within the context of a visual search, the dual-state model suggests that the target of the search will occupy the active position when it is variable (i.e., changes from trial to trial), and no interference from any accessory items will be evident. If, however, VWM resources are not required to support the constant updating of a search target, an accessory item may move into the active position and guide attention during search. Here we uncovered evidence that the target template does indeed occupy the active position (i.e., template-matching distractors are facilitated in search), but there was also evidence that additional (accessory) representations (i.e., the previewed distractors) were inhibited during search. Perhaps it is the case that, while only one representation can facilitate processing, multiple representations may guide inhibition during search, biasing attention away from goal-irrelevant information. Indeed, there is some evidence for an inhibitory role of accessory memory items (Downing \& Dodds, 2004). Specifically, Downing and Dodds noted that the presence of an accessory memory item in a visual search array resulted in enhanced target recognition (i.e., faster RTs), suggesting that the memory-matching stimulus was more easily ignored than other distractors.

An alternative explanation for our results, however, is that multiple items are capable of guiding attention, irrespective of VWM state (active vs. accessory) or attention type (facilitation vs. inhibition). Consistent with this alternative, Beck et al. (2012) demonstrated that visual search can be concurrently guided by two target templates in VWM, potentially indicating VWM-based facilitation by multiple representations. Those researchers examined eye movements in a visual search task and noted that, when selectively attending to only one of two colors in an array, observers searched consecutive items in one color before switching. This switch from one template to the next resulted in a switch cost (i.e., a time delay between fixation on the final item of the first attended color and fixation on the first item of the second attended color). Interestingly, when asked to attend to two colors at the same time, observers were able to switch their gaze back and forth between the two colors frequently, demonstrating no switch cost between items. It may, then, be possible for attentional selection to be driven by more than one template in VWM.

In summary, our results are incompatible with the claim that only one representation in VWM can guide selection at a time: How guidance by multiple representations is accomplished, however, remains an open question. Drawing from the dual-state model (Olivers et al., 2011), it may be the case that only one representation can guide selection through facilitation; however, this interpretation entails that accessory-state VWM representations can nevertheless concurrently guide selection through inhibition. Alternatively, our results are also consistent with the conclusion that multiple VWM representations can actively guide selection, through either facilitation or inhibition (Beck et al., 2012). Regardless, our results provide constraints on all models of VWM-based attention: Multiple VWM representations can concurrently guide selection through inhibition, and at the same time, a separate VWM representation can guide selection through facilitation.

\section{Spatial versus object working memory}

How might multiple VWM representations be capable of supporting both inhibition and facilitation simultaneously? One possible answer is that this ability is accomplished through separate spatial and object subdivisions of VWM, each of which is supported by a distinct neural substrate (see Luck, 2008). Whereas search target templates are typically defined by nonspatial features, and thus are likely predominantly supported by the object subdivision of VWM, the inhibition of previewed distractors could be accomplished on the basis of only spatial information, and the spatial 
subdivision. This account would help explain, for example, why search was guided by the full contents of memory in our tasks, but only by a single item in the tasks that support the dual-state model of VWM-based attention. According to this account, only a single representation in the object subdivision can support facilitation at a time (consistent with the dual-state model), whereas multiple spatial representations guide inhibition. Consistent with this explanation, Al-Aidroos et al. (2012) observed that individual differences in the capacity of preview inhibition correlated with a measure of spatial working memory, and not with color working memory. Although this account has some appeal, our assessment is that currently there are inadequate data to support it, and also there is reason to believe it is incorrect.

First, it is currently unclear whether spatial working memory alone supports the preview benefit reported here and in Al-Aidroos et al. (2012). Indeed, Al-Aidroos et al. were quite reserved in the conclusions that they drew from this finding, pointing out the methodological limitations of their color memory task. Furthermore, despite reports that object and spatial working memory may be segregated in posterior regions (Gnadt \& Andersen, 1988; Miller, Li, \& Desimone, 1993), the superior parietal lobe specifically subserves the preview effect (Allen, Humphreys, \& Matthews, 2008; Humphreys et al., 2004; Olivers, Smith, Matthews, \& Humphreys, 2005), and this region has been shown to be implicated in both spatial and object working memory (Xu \& Chun, 2006). Indeed, previous studies have demonstrated that changing the identities of the previewed distractors is disruptive to the preview benefit (Jiang, Chun, \& Marks, 2002; Kunar, Humphreys, Smith, \& Hulleman, 2003), and Emrich and colleagues (2010) have provided support for the involvement of nonspatial memory in inhibition during search. Thus, from this work alone, it is not clear that the spatial subsystem of VWM is what more heavily supports the preview benefit.

Despite the fact that the demands of a particular task will dictate whether spatial or nonspatial working memory is relied upon more heavily, the two are intricately linked, with some researchers suggesting that location is an obligatory feature of object memory (Jiang, Olson, \& Chun, 2000), and that in fact it is not necessary to regard spatial and nonspatial memory as separate systems (Rajsic \& Wilson, 2014). Moreover, independent of the debate regarding which working memory systems may or may not be involved in preview inhibition, it remains the case that the present data demonstrate concurrent guidance of attention by multiple VWM representations - a point that adds constraints to existing models of the VWM-based guidance of attention. The question of how different VWM subsystems contribute to attentional guidance in dual-state models remains an open question.

\section{Comparing preview search and visual search}

To what extent can we generalize conclusions drawn from a preview search paradigm to more conventional, nonpreview visual searches? In the present experiments, we used the preview paradigm to directly control which search distractors participants processed first, and measured whether these specific distractors were successfully inhibited. Presenting a subset of search distractors before the rest of the search display, however, creates differences between the previewed and nonpreviewed distractors, such as temporal discontinuities (Jiang et al., 2002) and differences in physical salience (Donk \& Theeuwes, 2001, 2003). Do the present results regarding the VWM-based inhibition of previewed distractors tell us about how distractors are inhibited in nonpreview searches?

There is both neurophysiological and behavioral evidence to suggest that, beyond previewed distractors, VWM also supports the inhibition of nonpreviewed distractors. The CDA, an ERP associated with VWM maintenance, has been observed while participants completed a visual search task (Emrich et al., 2009). In this study, the visual search target was static throughout the duration of the experiment, and thus the CDA activity cannot be attributed to the maintenance of a target template. Moreover, the magnitude of this component, which is associated with the amount of information stored in VWM, increased relatively slowly after the onset of the search display, and took longer to reach peak amplitude in participants with greater VWM capacities, consistent with the interpretation that as participants reject search distractors, these distractors are encoded in memory until capacity is reached. To further examine the involvement of VWM in search, Emrich and colleagues (2010) manipulated the extent to which VWM resources were available to an observer while performing visual search, by including a concurrent VWM change detection task. As would be expected if VWM supports the inhibition of a small number of rejected search distractors, increases in the concurrent memory load caused increases in overall search times, and not in search slopes. This effect was proportionate to the memory load until VWM capacity was reached - when observers were required to maintain more than four items, no further increases in search RTs were observed. These two studies suggest that VWM-based inhibition does not operate only on previewed distractors, and that the observation of simultaneous guidance of inhibition and facilitation by VWM in the present study generalizes to more conventional, nonpreview searches.

\section{Inhibition of eye movements or perceptual processing?}

The present tasks were designed to encourage participants to make eye movements during their visual searches. Notably, when search can be completed covertly (i.e., without eye 
movements), the signature of VWM-based inhibition is not evident (see Al-Aidroos et al., 2012, Exp. 2B). Might this pattern indicate that VWM-based inhibition only influences where participants look, or does VWM-based inhibition directly influence covert spatial attention?

Although the results of Al-Aidroos et al. (2012) may suggest that any effect of VWM-based inhibition on perceptual processing is an indirect consequence of its effects on eye movements, their findings do not speak to this issue as clearly as it may seem. Numerous mechanisms allow observers to prioritize new information over old (Donk, 2006; Olivers et al., 2006), and the constraints of a task determine which of these mechanisms will ultimately guide behavior. In particular, when preview searches are completed covertly, the preview effect tends to be dominated by a highly effective, seemingly unlimited-capacity mechanism (Al-Aidroos et al., 2012, Exp. 2B). The observed preview benefit in such searches is likely guided, in part, by attentional capture from the onset of nonpreviewed search items (Donk \& Theeuwes, 2001) and by other potentially capacity-unlimited inhibitory mechanisms (von Mühlenen, Watson, \& Gunnell, 2013). The contribution of VWM-based preview inhibition can only be observed in measures of behavioral performance when other preview mechanisms are prevented from guiding the search process (e.g., by allowing participants to move their eyes, which disrupts the sensory signals that capture attention). Accordingly, it remains possible that VWM-based inhibition has a direct influence on covert spatial attention. Indeed, the electrophysiological results of Emrich and colleagues (2009) provide evidence that VWM-based inhibition contributes to search efficiency even when participants complete searches without eye movements. Although further investigation will be required to confirm whether the effect is direct or indirect, what is clear from the present study is that VWM representations can be used to simultaneously guide perceptual processing through both inhibition and facilitation.

\section{Concluding remarks}

The results reported here provide evidence that VWM-based preview inhibition (i.e., the marking and subsequent inhibition of previewed distractors) during search remains observable even when VWM resources are also required to maintain the identity of a search target. These results advance our understanding of the limits on interactions between VWM and attention. Whereas previous studies have observed that only one activated representation in VWM can guide perceptual selection, here we showed that under some circumstances multiple representations can guide attention at the same time. Moreover, VWM is capable of simultaneously guiding two seemingly opposing processes: the facilitation of a target and the inhibition of distractors. Taken together, these findings indicate that the full contents of VWM can flexibly, and simultaneously, bias the selection of visual information, influencing the efficiency of visual search.

Author note This work was supported by a Natural Sciences and Engineering Research Council (NSERC) Discovery Grant to N.A., and by an NSERC Canada Graduate Scholarship to B.D.

\section{References}

Al-Aidroos, N., Emrich, S. M., Ferber, S., \& Pratt, J. (2012). Visual working memory supports the inhibition of previously processed information: Evidence from preview search. Journal of Experimental Psychology: Human Perception and Performance, 38, 643-663. doi:10.1037/a0025707

Allen, H. A., Humphreys, G. W., \& Matthews, P. M. (2008). A neural marker of content-specific active ignoring. Journal of Experimental Psychology: Human Perception and Performance, 34, 286-297.

Alvarez, G. A., \& Cavanagh, P. (2004). The capacity of visual short-term memory is set both by visual information load and by number of objects. Psychological Science, 15, 106-111. doi:10.1111/j.09637214.2004.01502006.x

Baddeley, A. (1986). Working memory. Oxford: Oxford University Press, Clarendon Press.

Bays, P. M., Catalao, R. F. G., \& Husain, M. (2009). The precision of visual working memory is set by allocation of a shared resource. Journal of Vision, 9(10), 1-11. doi:10.1167/9.10.7

Bays, P. M., \& Husain, M. (2008). Dynamic shifts of limited working memory resources in human vision. Science, 321, 851-854. doi:10. $1126 /$ science. 1158023

Beck, V. M., Hollingworth, A., \& Luck, S. J. (2012). Simultaneous control of attention by multiple working memory representations. Psychological Science, 23, 887-898. doi:10.1177/0956797612439068

Belopolsky, A. V., \& Theeuwes, J. (2009). Inhibition of saccadic eye movements to locations in spatial working memory. Attention, Perception, \& Psychophysics, 71, 620-631. doi:10.3758/APP.71.3. 620

Brockmole, J. R., \& Henderson, J. M. (2005). Prioritization of new objects in real-world scenes: Evidence from eye movements. Journal of Experimental Psychology: Human Perception and Performance, 31, 857-868. doi:10.1037/ 0096-1523.31.5.857

Bundesen, C. (1990). A theory of visual attention. Psychological Review, 97, 523-547. doi:10.1037/0033-295X.97.4.523

Carlisle, N. B., Arita, J. T., Pardo, D., \& Woodman, G. F. (2011). Behavioral/systems/cognitive attentional templates in visual working memory. Journal of Neuroscience, 31, 9315-9322. doi:10.1523/ JNEUROSCI.1097-11.2011

Castel, A. D., Pratt, J., \& Craik, F. I. M. (2003). The role of spatial working memory in inhibition of return : Evidence from divided attention tasks. Perception \& Psychophysics, 65, 970-981. doi:10. 3758/BF03194827

Chelazzi, L., Duncan, J., Miller, E. K., \& Desimone, R. (1998). Responses of neurons in inferior temporal cortex during memory-guided visual search. Journal of Neurophysiology, 80, 2918-2940. doi:10.1111/j.1559-1816.2002.tb00236.x

Chelazzi, L., Miller, E., Duncan, J., \& Desimone, R. (1993). A neural basis for visual search in inferior temporal cortex. Nature, 363, 345-347. doi:10.1038/363345a0

Cohen, A., \& Ivry, R. (1989). Illusory conjunctions inside and outside the focus of attention. Journal of Experimental 
Psychology: Human Perception and Performance, 15, 650-663. doi:10.1037/0096-1523.15.4.650

Cohen, A., \& Ivry, R. B. (1991). Density effects in conjunction search: Evidence for a coarse location mechanism of feature integration. Journal of Experimental Psychology: Human Perception and Performance, 17, 891-901. doi:10.1037/0096-1523.17.4.891

Cousineau, D. (2005). Confidence intervals in within-subject designs: A simpler solution to Loftus and Masson's method. Tutorials in Quantitative Methods for Psychology, 1, 42-45.

Cowan, N. (2001). The magical number 4 in short-term memory: A reconsideration of mental storage capacity. Behavioral and Brain Sciences, 24, 87-114. doi:10.1017/S0140525X01003922

Dalvit, S., \& Eimer, M. (2011). Memory-driven attentional capture is modulated by temporal task demands. Visual Cognition, 9, 145-153. doi:10.1080/13506285.2010.543441

Desimone, R., \& Duncan, J. S. (1995). Neural mechanisms of selective visual attention. Annual Review of Neuroscience, 18, 193-222. doi: 10.1146/annurev.ne.18.030195.001205

Donk, M. (2006). The preview benefit: Visual marking, feature-based inhibition, temporal segregation, or onset capture? Visual Cognition, 14, 736-748. doi:10.1080/13506280500193230

Donk, M., \& Theeuwes, J. (2001). Visual marking beside the mark: Prioritizing selection by abrupt onsets. Perception \& Psychophysics, 63, 891-900. doi:10.3758/BF03194445

Donk, M., \& Theeuwes, J. (2003). Prioritizing selection of new elements: Bottom-up versus top-down control. Perception \& Psychophysics, 65, 1231-1242.

Downing, P. E., \& Dodds, C. M. (2004). Competition in visual working memory for control of search. Visual Cognition, 11, 689-703. doi: $10.1080 / 13506280344000446$

Duncan, J., \& Humphreys, G. W. (1989). Visual search and stimulus similarity. Psychological Review, 96, 433-458. doi:10.1037/0033295X.96.3.433

Eimer, M., \& Kiss, M. (2010). Top-down search strategies determine attentional capture in visual search: Behavioral and electrophysiological evidence. Attention, Perception, \& Psychophysics, 72, 951-962. doi:10.3758/APP.72.4.951

Emrich, S. M., Al-Aidroos, N., Pratt, J., \& Ferber, S. (2009). Visual search elicits the electrophysiological marker of visual working memory. PLoS ONE, 4, e8042. doi:10.1371/journal. pone. 0008042

Emrich, S. M., Al-Aidroos, N., Pratt, J., \& Ferber, S. (2010). Finding memory in search: The effect of visual working memory load on visual search. Quarterly Journal of Experimental Psychology, 63, 1457-1466. doi:10.1080/17470218.2010.483768

Emrich, S. M., Ruppel, J. D. N., Al-Aidroos, N., Pratt, J., \& Ferber, S. (2008). Out with the old: Inhibition of old items in a preview search is limited. Perception \& Psychophysics, 70, 1552-1557. doi:10. 3758/PP.70.8.1552

Gnadt, J. W., \& Andersen, R. A. (1988). Memory related motor planning activity in posterior parietal cortex of macaque. Experimental Brain Research, 70, 216-220.

Houtkamp, R., \& Roelfsema, P. R. (2006). The effect of items in working memory on the deployment of attention and the eyes during visual search. Journal of Experimental Psychology: Human Perception and Performance, 32, 423-442. doi:10.1037/0096-1523.32.2.423

Humphreys, G. W., Kyllingsbaek, S., Watson, D. G., Olivers, C. N. L., Law, I., \& Paulson, O. B. (2004). Parieto-occipital areas involved in efficient filtering in search: A time course analysis of visual marking using behavioural and functional imaging procedures. Quarterly Journal of Experimental Psychology, 57, 610-635. doi:10.1080/02724980343000620

Jiang, Y., Chun, M. M., \& Marks, L. E. (2002). Visual marking: Selective attention to asynchronous temporal groups. Journal of Experimental Psychology: Human Perception and Performance, 28, 717-730. doi:10.1037/0096-1523.28.3.717
Jiang, Y., Olson, I. R., \& Chun, M. M. (2000). Organization of visual short-term memory. Journal of Experimental Psychology: Learning, Memory, and Cognition, 26, 683-702. doi:10.1037/0278-7393.26. 3.683

Keshvari, S., van den Berg, R., \& Ma, W. J. (2013). No evidence for an item limit in change detection. PLoS Computational Biology, 9, e1002927. doi:10.1371/journal.pcbi.1002927

Kunar, M. A., Humphreys, G. W., Smith, K. J., \& Hulleman, J. (2003). What is "marked" in visual marking? Evidence for effects of configuration in preview search. Perception \& Psychophysics, 65, 982-996. doi:10.3758/BF03194828

Luck, S. J. (2008). Visual short-term memory. In S. J. Luck \& A. Hollingworth (Eds.), Visual memory (pp. 43-86). New York, NY: Oxford University Press.

Luck, S. J., \& Vogel, E. K. (1997). The capacity of visual working memory for features and conjunctions. Nature, 390, 279-281. doi: $10.1038 / 36846$

Miller, E. K., Li, L., \& Desimone, R. (1993). Activity of neurons in anterior inferior temporal cortex during a short-term memory task. Journal of Neuroscience, 13, 1460-1478.

Olivers, C. N. L. (2009). What drives memory-driven attentional capture? The effects of memory type, display type, and search type. Journal of Experimental Psychology: Human Perception and Performance, 35, 1275-1291. doi:10.1037/a0013896

Olivers, C. N. L., \& Eimer, M. (2011). On the difference between working memory and attentional set. Neuropsychologia, 49, 1553-1558. doi:10.1016/j.neuropsychologia.2010.11.033

Olivers, C. N. L., Humphreys, G. W., \& Braithwaite, J. J. (2006). The preview search task: Evidence for visual marking. Visual Cognition, 14, 716-735. doi:10.1080/13506280500194188

Olivers, C. N. L., Meijer, F., \& Theeuwes, J. (2006). Featurebased memory-driven attentional capture: Visual working memory content affects visual attention. Journal of Experimental Psychology: Human Perception and Performance, 32, 1243-1265. doi:10.1037/0096-1523.32.5. 1243

Olivers, C. N. L., Peters, J., Houtkamp, R., \& Roelfsema, P. R. (2011). Different states in visual working memory: When it guides attention and when it does not. Trends in Cognitive Sciences, 15, 327-334. doi:10.1016/j.tics.2011.05.004

Olivers, C. N. L., Smith, S., Matthews, P. M., \& Humphreys, G. W. (2005). Prioritizing new over old: An fMRI study of the preview search task. Human Brain Mapping, 24, 69-78.

Pashler, H. (1987). Target-distractor discriminability in visual search. Perception \& Psychophysics, 41, 285-292. doi:10.3758/ BF03208228

Peters, J. C., Goebel, R., \& Roelfsema, P. R. (2009). Remembered but unused: The accessory items in working memory that do not guide attention. Journal of Cognitive Neuroscience, 21, 1081-1091. doi: 10.1162 /jocn.2009.21083

Rajsic, J., \& Wilson, D. E. (2014). Asymmetrical access to color and location in visual working memory. Attention, Perception, \& Psychophysics, 76, 1902-1913. doi:10.3758/s13414-014-0723-2

Sawaki, R., \& Luck, S. J. (2011). Active suppression of distractors that match the contents of visual working memory. Visual Cognition, 19, 956-972. doi:10.1080/13506285.2011.603709

Soto, D., Heinke, D., Humphreys, G. W., \& Blanco, M. J. (2005). Early, involuntary top-down guidance of attention from working memory. Journal of Experimental Psychology: Human Perception and Performance, 31, 248-261. doi:10.1037/0096-1523.31.2.248

Soto, D., Hodsoll, J., Rotshtein, P., \& Humphreys, G. W. (2008). Automatic guidance of attention from working memory. Trends in Cognitive Sciences, 12, 342-348. doi:10.1016/j.tics.2008.05.007

Theeuwes, J., Olivers, C. N. L., \& Chizk, C. L. (2005). Remembering a location makes the eyes curve away. Psychological Science, 16, 196-199. doi:10.1111/j.0956-7976.2005.00803.x 
Vogel, E. K., \& Machizawa, M. G. (2004). Neural activity predicts individual differences in visual working memory capacity. Nature, 428 , 748-751. doi: $10.1038 /$ nature 02447

von Mühlenen, A., Watson, D. G., \& Gunnell, D. (2013). Blink and you won't miss it: The preview benefit in visual marking survives internally generated eye blinks. Journal of Experimental Psychology: Human Perception and Performance, 39, 1279-1290.

Watson, D. G., \& Humphreys, G. W. (1997). Visual marking: Prioritizing selection for new objects by top-down attentional inhibition of old objects. Psychological Review, 104, 90-122. doi:10.1037/0033295X.104.1.90

Watson, D. G., \& Humphreys, G. W. (2005). Visual marking: The effects of irrelevant changes on preview search. Perception \& Psychophysics, 67, 418-434.
Wolfe, J. M. (1994). Guided Search 2.0: A revised model of visual search. Psychonomic Bulletin \& Review, 1, 202-238. doi:10.3758/ BF03200774

Woodman, G. F., \& Arita, J. T. (2011). Direct electrophysiological measurement of attentional templates in visual working memory. Association for Psychological Science, 22, 212-215.

Woodman, G. F., \& Luck, S. J. (2007). Do the contents of working memory automatically influence attentional selection during visual search? Journal of Experimental Psychology: Human Perception and Performance, 33, 363-377. doi:10.1037/0096-1523.33.2.363

Woodman, G. F., Luck, S. J., \& Schall, J. D. (2007). The role of working memory representations in the control of attention. Cerebral Cortex, 17, 118-124. doi:10.1093/cercor/bhm065

Xu, Y., \& Chun, M. M. (2006). Dissociable neural mechanisms supporting visual short-term memory for objects. Nature, 440, 91-95. doi:10.1038/nature04262 